\title{
Le passage du jeu à la création : le cas du jeu vidéo amateur
}

\section{Pierre-Yves Hurel}

\section{OpenEdition}

1 Journals

Édition électronique

URL : http://journals.openedition.org/sdj/766

DOI : $10.4000 /$ sdj.766

ISSN : 2269-2657

\section{Éditeur}

Laboratoire EXPERICE - Centre de Recherche Interuniversitaire Expérience Ressources Culturelles Education

\section{Référence électronique}

Pierre-Yves Hurel, «Le passage du jeu à la création : le cas du jeu vidéo amateur », Sciences du jeu [En ligne], 7 | 2017, mis en ligne le 19 février 2017, consulté le 28 mars 2021. URL : http:// journals.openedition.org/sdj/766 ; DOI : https://doi.org/10.4000/sdj.766

Ce document a été généré automatiquement le 28 mars 2021.

\section{(c) $(1) \Theta \Theta$}

La revue Sciences du jeu est mise à disposition selon les termes de la Licence Creative Commons Attribution - Pas d'Utilisation Commerciale - Pas de Modification 4.0 International. 


\title{
Le passage du jeu à la création : le cas du jeu vidéo amateur
}

\author{
Pierre-Yves Hurel
}

La création de jeux vidéo en amateur est une pratique conséquente sur le plan quantitatif et hétérogène sur le plan qualitatif. Nous nous limiterons ici à donner quelques indicateurs liés à la pratique reposant sur l'utilisation de logiciels d'aide à la programmation, objet principal de notre travail de doctorat. Sur le plan quantitatif d'abord, les chiffres décrivent une activité importante : créé par Damien Djaouti (2011) dans le cadre de sa thèse de doctorat, le site internet collaboratif gameclassification.com recense 484 programmes et éditeurs de niveaux sortis depuis 1980 (tous les chiffres de cette section sont issus d'une consultation des sites cités au 01-06-2016). Parmi ces logiciels, les plus ambitieux (sur le plan commercial) sont accompagnés de forums officiels et les plus populaires sont l'objet de l'attention de forums officieux créés à l'initiative de leurs utilisateurs. Par exemple, le forum officiel de Scirra (logiciels Construct Classics et Construct 2) compte 237125 utilisateurs enregistrés sur le forum et 6121 jeux publiés. Le pendant officieux francophone, la communauté Construct-French, compte quant à elle 740 membres.

2 Liés à un seul programme informatique, ces quelques chiffres prouvent l'existence d'un milieu vaste, fourmillant et productif qui ne peut s'expliquer uniquement par la présence de professionnels parmi ces utilisateurs. En dehors de ces logiciels de création, il faut par ailleurs ajouter le secteur de la modification logicielle de jeux existants (le modding), la création sur des plateformes propriétaires (le homebrew) et une partie de la programmation libre.

3 La pratique se révèle tout aussi intéressante sur le plan qualitatif : ces logiciels d'aide proposent quasiment tous un rapport spécifique à la création. Damien Djaouti, distingue les logiciels généralistes (Unity, Game Maker, Unreal Engine, etc.) et ceux spécialisés dans un genre particulier (RPG Maker, Adventure Game Studio, Twine, etc.). Nous pourrions distinguer parmi eux ceux qui recourent à des langages de programmation (Unity, Pico8, etc.) et ceux qui remplacent la programmation par une interface visuelle (Construct 2, Scratch, etc.). Remarquons aussi que certains 
programmes sont dédiés à une franchise (tels que Zquest, un logiciel amateur pour créer son propre Zelda).

4 Les pratiques elles-mêmes sont variées. On trouve aussi bien sur les communautés des projets menés en solitaire ou en équipe depuis dix ans que des gamejams limitées à 24 ou 48 heures. Certains sont des fangames prenant pour objet des franchises comme Final Fantasy (mentionnons Final Fantasy VII Origin - toutes les productions d'amateurs citées à titre d'exemples sont issues de communautés dédiées à RPG Maker et peuvent être consultées sur rpg-maker.fr) ou The Legend of Zelda (par exemple Zelda Mystery of Solarus), alors que d'autres reposant sur un univers original (citons la série des Laxius Power). D'autres œuvres encore prennent les communautés pour objet afin d'affirmer et actualiser l'existence d'un groupe, d'un «nous » (voir la série des Rutipa's Quest). Notons enfin que l'on y trouve des pratiques connexes variées, telles que les concours consacrant les meilleures créations de la communauté, mais aussi des news, tests, interviews, archives et tutoriels.

5 Ce champ a été assez peu investi par les études académiques malgré l'intérêt que peut susciter l'appropriation d'un média longtemps perçu comme étant trop coûteux (en temps, en compétences, en investissement) pour être réalisable en dehors des circuits commerciaux. Nous pouvons noter tout de même un intérêt marqué pour les mods, principalement étudiés sous l'angle de la professionnalisation des acteurs et autres points de jonction avec l'industrie vidéoludique (Postigo, 2007; Sotamaa, 2010); ainsi que pour les utilisateurs de logiciels d'aide à la création dans l'optique de trouver, dans ces situations d'apprentissages informels, des facilitateurs à mettre au service de programmes éducatifs (Gee and Tran, 2016 ; Burke and Kafai, 2014 ; Owens, 2013).

6 Ce type de pratique n'est généralement étudié que lorsqu'il est perçu comme étant source d'une plus-value pour l'industrie ou l'éducation. La domination du paradigme de la convergence (Jenkins, 2006 ; Flichy, 2010) au sein des études des fans (fans studies), qui se concentrent sur les nouvelles modalités de rencontre industries-publics, est un facteur explicatif possible de ce phénomène. La focalisation sur ce point de jonction répond effectivement à des enjeux contemporains importants, mais elle ne permet pas de penser les pratiques autrement que sous l'angle (politique) de l'amateur dont la production peut être mise à profit ou du militant qui entrerait au contraire en résistance grâce à sa pratique créative. C'est pourquoi nous adopterons une perspective s'inscrivant plutôt dans le champ des pratiques culturelles, à l'instar des études de musiciens amateurs (Dubois, Méon et Pierru, 2010), de cinéastes amateurs (Odin, 1999), et plus globalement de l'amateurisme (Hennion, 2009).

7 Dans cet article, nous proposons d'interroger les liens entre l'activité de jouer à un jeu vidéo et celle d'en créer un (dans un cadre amateur) en tant que passages de frontière. Est-ce que créer un jeu revient à jouer? Que peut bien signifier "prolonger » le jeu? Qu'est-ce qui est prolongé dans ce cas-là ? Quel est le statut d'une œuvre produite par un créateur qui se définit avant tout comme un joueur? Nous verrons que, au sein de la production en amateur, le jeu est à la fois central (en tant que plaisir et goût) et à la marge (en tant que pratique prenant place avant ou après l'activité de création). Sur la base des différentes dynamiques de création mises à jour au cours de l'article, nous proposerons enfin d'introduire la notion d'amateurisme dans le champ des sciences du jeu en tant qu'articulation conceptuelle nécessaire pour comprendre la question du prolongement. 


\section{Méthodologie : une approche par théorisation ancrée}

8 Notre travail de recherche, qui suit les principes de la théorisation ancrée, propose de mettre de côté les cadres conceptuels préexistants qui pourraient être utilisés pour tenter d'expliquer la pratique étudiée. Notre objectif est de donner la priorité à la parole des amateurs et de co-construire avec eux un cadre conceptuel. Ce choix est aussi politique : il répond à la domination symbolique que peut représenter l'absence de cette parole tant dans le domaine académique que dans la presse spécialisée en jeux vidéo (à l'exception de l'histoire récurrente de « cet amateur devenu professionnel »). Cette absence constitue une mise au ban des amateurs de la culture vidéoludique (Shaw, 2010). Notre objectif est d'avancer par triangulation (Héas et Poutrain, 2003) vers une compréhension des pratiques, des vécus, des postures et des étiquettes en associant différents moyens de récolte et de production de données.

En cours de réalisation, notre enquête compte à ce stade une douzaine d'entretiens compréhensifs. Les enquêtés sont Belges (7), Français (4) et Canadiens (Québec) (1), ont entre 25 et 42 ans et sont tous des hommes. Ils ont été recrutés sur base de leur position centrale dans des communautés et/ou une longue expérience en tant qu'utilisateurs de logiciels d'aide à la création. Ils utilisent RPG Maker (8), Construct 2 (2) et Unity (2). Ces entretiens ont été réalisés en présentiel (8) et via des messageries instantanées (4). L'échantillon sera diversifié dans la suite de l'enquête (sur tous les aspects : genre, âge, positions dans les communautés, etc.). C'est principalement sur des extraits de ces entretiens et sur quelques citations issues de forums en ligne que nous nous baserons dans le cadre de cet article. A ce stade de la recherche, il ne nous est pas encore possible de présenter un cadre théorique. Nous proposerons plutôt d'explorer ici quelques premiers résultats issus du terrain.

\section{Les jeux vidéo imaginaires : le prolongement par la feintise ludique}

10 Lors des entretiens, notre première question consiste à demander comment l'enquêté «en est venu à créer des jeux». Alors que l'on pouvait s'attendre à une histoire débutant lors de la découverte des logiciels de création, les enquêtés commencent souvent leur explication (10 fois sur les 12) en se référant à des pratiques datant de leur enfance (généralement vers 9-12 ans). Ils sont d'abord des joueurs: ils jouent " énormément », « depuis toujours », c'est « la » grande passion. « Et puis », un jour ils ont eu l'envie de créer des jeux. Stéphane (les prénoms des enquêtés ont été modifiés) raconte: «Quand j'étais petit [...] je mettais des feuilles bout à bout [pour faire] des niveaux, un peu à la Mario. J'avais un petit perso [...] dans un écran en carton, avec des manettes en carton et puis je faisais défiler ça dans l'écran, j'essayais de simuler un jeu vidéo $[. .]$.$» .$

11 Ce type d'anecdote fondatrice et récurrente peut prendre des formes matérielles très différentes. Victor explique par exemple qu'il a « entrepris à maintes reprises de faire des univers et des histoires de plus en plus abouties (au tout début avec [ses] jouets en imitant Star Wars, Zelda \& cie), et [qu'il en est] arrivé naturellement à la création d'un RPG [...]». D'autres enquêtés nous racontent avoir «fait comme si [il créait] des jeux vidéo mais avec des Lego », ou encore avoir dessiné des "Zelda sur papier » pour faire 
jouer un frère ou un ami. Différents aspects de ces témoignages sont communément partagés et permettent de dégager dans quelles conditions leur pratique émerge.

D'abord, les enquêtés font référence systématiquement à des franchises particulières. Ainsi, ils expliquent avoir créé "des Zelda sur papier ", " des Metroid en Lego », des "Mario en carton ». Cette transposition de jeux vidéo dans des contextes différents se présente comme une situation dans laquelle les enquêtés manient les codes des œuvres consommés. Cédric raconte avoir créé des "donjons de Zelda sur papier ", des sortes de "labyrinthes améliorés ». Il indiquait à son petit frère sa position dans le niveau et lui disait ce qu'il pouvait faire, le résultat de ses actions, etc. Ils jouent alors avec l'univers fictionnel autant dans sa dimension narrative (s'inspirant des éléments thématiques) que ludique (transposant des règles de jeux à d'autres contextes).

Ensuite, les enquêtés expriment régulièrement une posture fictionnelle ou de second degré lorsqu'ils tentent de "simuler " un jeu, ce que d'autres vont exprimer comme étant des " jeux vidéo imaginaires", "des faux jeux vidéo", une façon de "faire comme si » ils créaient des œuvres, ou lorsqu'ils décrivent une "sorte de rêve de créer un jeu ». En d'autres termes, ces témoignages nous semblent montrer qu'à ce stade, et sans présager de la suite de la pratique, les enquêtés sont passés à un autre type de jeu, relevant de la feintise ludique (Schaeffer, 1999) ou du masque (Caillois, 1992): ils feignent de créer des jeux vidéo.

De plus, l'envie de créer est présente chez ces enquêtés avant d'avoir trouvé les moyens de leurs ambitions. Ce désir est d'ailleurs souvent synonyme de frustrations intenses: « tout était en carton, on ne pouvait pas vraiment jouer, donc en fait c'était nul ! C'était très frustrant ! », raconte Stéphane. Nous comprenons dans ces conditions l'excitation vécue lors de la découverte d'un logiciel : "Au début j'ai cru que c'était une arnaque » dit Stéphane. "Quand j'ai compris que c'était faisable, je me suis dit qu'il fallait que j'apprenne ça tout de suite ", raconte Cédric. Ceci vient contrer d'emblée d'éventuelles tentations déterministes sur le plan technologique : la présence des programmes à elle seule n'explique pas l'existence de la pratique. Les logiciels ne produisent pas l'envie de créer des jeux vidéo, ils la rencontrent.

La mise en récit produite dans les entretiens vient désigner la pratique de l'utilisation des logiciels comme le prolongement de ces pratiques fantasmatiques et ludiques précédentes, elles-mêmes présentées comme étant la suite logique du fait de jouer. Ces activités peuvent être abordées comme un prolongement du jeu, au même titre que les fanfictions. Mais qu'est-ce qui est prolongé exactement dans cette situation? Concrètement, la pratique en elle-même s'arrête et l'enquêté sort de ce jeu-là pour que puisse prendre place une autre pratique ludique, créative et référentielle. Ce qui est prolongé, ce n'est pas tant le jeu en lui-même que le plaisir qu'il procure. Nous reviendrons sur ce processus par la suite.

\section{La rencontre usager-logiciel : de l'appropriation ludique}

Les logiciels tels que RPG Maker sont nommés de différentes manières en français comme en anglais : « logiciels » ou " programs ", « outils » ou « tools", « moteurs de jeu » ou «engines». Damien Djaouti $(2011$, p. 49, 200) distingue les « usines à jeux » (les logiciels accessibles qui produisent des œuvres auxquelles on peut jouer 
indépendamment du programme original), les « middlewares » (les outils de création qui demandent une connaissance technique poussée) et les «jeux 2.0" (les jeux qui consistent à créer des niveaux de jeu, tels que Mario Maker ou Little Big Planet). Les termes employés pour désigner les logiciels se réfèrent généralement à des moyens d'action (« outils », « moteur ») voire au travail (« usine à jeu »).

Pourtant, une partie de nos enquêtés désignent ces outils comme des "jouets", des "jeux pour créer des jeux», des "joujoux». Victor, rapporte que «certains considèrent qu'on ne développe pas sur RPG Maker mais qu'on joue à RPG Maker (...). » Il ajoute que l'une de ses connaissances s'en explique en citant le slogan officiel du programme : "Assez simple pour un enfant, assez puissant pour un développeur de jeux. » ${ }^{1}$ Selon lui «à la base [RPG Maker] était plutôt vu par les créateurs comme un jeu, c'est quand ils ont vu que certains s'en servaient sérieusement qu'ils ont fait de la pub là-dessus." Nous reviendrons plus tard sur l'usage du terme "sérieusement ". Globalement, la plupart des enquêtés désignent leur activité comme "amusante ", voire «ludique ». Nous interrogerons ici cette distinction logiciels/jeux/jouets en tant que témoin d'une appropriation ludique du dispositif.

avons exposé précédemment la filiation entre des pratiques créatives et enfantines et l'utilisation des outils en question. Comme nous l'avons vu, la production de jeux était alors un jeu en soi qui consistait à feindre la création, faute de moyens techniques. Que se passe-t-il lorsque la création est enfin à la portée des enquêtés? Au moins le temps d'utiliser le logiciel, il faut que la pratique initiale de la feintise cesse. Certains commencent alors à « jouer » avec le, voire au, logiciel. Les deux prépositions révèlent deux postures rhétoriques qui répondent à deux attitudes ludiques observées.

Celui qui joue avec le logiciel le considère comme un jouet. Il teste, il expérimente, il prend plaisir à se faire surprendre par des réactions inattendues du programme lors des phases de tests, bref, il «fait n'importe quoi avec», comme l'expliquent certains enquêtés. Une première façon de construire sa relation au dispositif se fait sur le mode $\mathrm{du}$ jouet et de l'attitude ludique qui en découle: celle de la recherche des possibles ludiques. La seconde approche consiste à construire les dispositifs comme des jeux, indiquant un but. Il s'agit alors de considérer que le «but » de RPG Maker est de créer des RPG "comme son nom l'indique». Ainsi, l'utilisateur va chercher à remplir l'objectif : créer un RPG japonais classique, répondant aux normes du genre. Cette interprétation est probablement plus encline à se développer chez les utilisateurs de logiciels spécialisés dans un genre ou une franchise spécifique. Cette première phase d'appropriation ludique, sous une de ses deux modalités, apparaît comme étant commune aux utilisateurs lors des premiers temps de l'utilisation du logiciel, puis perdure pour une partie d'entre eux.

\section{Le déplacement de la production du plaisir : jouer du jeu vidéo}

De manière quasiment généralisée, les acteurs du terrain (en entretiens et sur les forums) ne disent pas « je crée un jeu » mais « je crée mon jeu », ou « mon RPG», voire «mon Zelda ». L'expression dénote un sentiment d'appartenance : la création est la sienne, quand bien même il s'agirait d'un fangame ou d'une production réalisée en grande partie avec l'aide d'un outil. L'inscription dans une culture du « remix » et dans 
l'amateurisme ne signifie pas pour autant l'abandon de toute revendication de la paternité de sa création. D'ailleurs, lorsque des images créées par d'autres utilisateurs sont remixées, l'usage est de citer l'auteur dans les crédits.

L'expression désigne en même temps le caractère personnel de la création: "mon jeu ", c'est celui qui exprime mes goûts en tant que joueur. Lorsqu'ils découvrent le ou les logiciels qu'ils vont adopter, les enquêtés ne deviennent pas subitement des artistes ou des auteurs du simple fait de leur passage à la création. Et, bien qu'ils s'approprient le logiciel sur le mode du jeu, leur activité continue de se référer à leurs goûts en tant que joueurs - tout comme ils le faisaient avec leurs «jeux imaginaires». Ainsi, un utilisateur de RPG Maker, Victor, nous rapporte des «stimulations similaires » vécues pendant l'acte de jouer et celui de créer: "par exemple, en paramétrant des statistiques d'équipements, en cachant des coffres dans les décors, je ressens un plaisir fort similaire à celui de découvrir des équipements et des coffres cachés dans un RPG [...] ». L'anecdote est illustrative de la tendance générale : les amateurs qui cherchent à prendre du plaisir en créant se basent en effet généralement sur leurs propres goûts et expériences de jeux.

Il semblerait que nos enquêtés aient déplacé la production du plaisir issu de l'utilisation des œuvres à celle découlant de l'utilisation des outils de création. Ils ne jouent plus aux jeux vidéo, ils jouent $d u$ jeu vidéo à travers les logiciels que nous proposons dès lors de constituer comme des «instruments de jeu vidéo». La guitare et la flûte sont deux instruments de musique qui permettent des pratiques différentes et sous-tendent des logiques de production du son à la fois liées et distinctes (nous limitons l'usage de cette métaphore à ce qui relève de la pratique créative, et non aux différences constitutives du son ou du jeu produit en fonction de l'instrument utilisé). Cette logique est partagée par les instruments de jeu vidéo : les œuvres créées à travers RPG Maker sont le fruit d'expériences dissemblables de ceux créés avec Unity ou Construct 2 : les interfaces sont (parfois radicalement) différentes.

23 L'utilisateur de Construct 2 va commencer par intégrer des images, puis va être très vite confronté au besoin de "coder" à travers les menus de conditions et actions. L'utilisateur de RPG Maker aura d'emblée à manipuler une carte du monde, qu'il pourra "dessiner» à travers l'assemblage de tuiles de dessins préexistantes (et personnalisables). La partie "programmation » elle-même sera en grande partie dépendante de la carte du jeu créé : l'utilisateur place des « évènements » sur des cases de la carte, etc. De manière plus générale, lorsqu'il rapporte certains résultats de son auto-ethnographie en tant qu'utilisateur de Unity, Roth (2015) identifie deux caractéristiques du logiciel comme autant d'invitations au jeu : d'abord la possibilité de « remixer » des éléments créés par d'autres utilisateurs, ensuite la possibilité de tester quasiment instantanément le jeu en cours de création - et les surprises qui peuvent en découler.

Nous avons vu que les enquêtés construisaient en partie leur relation aux logiciels sur le mode du jeu (en termes d'appropriation ludique mais aussi de reconstruction d'une expérience passée de joueur). On peut, en renversant la perspective, considérer que ces outils sont en quelque sorte «jouables» (Genvo, 2012): ils présentent des caractéristiques susceptibles de favoriser leur adaptation à une attitude ludique. Dans le cadre de la création en amateur, l'utilisation d'un logiciel de création de jeux vidéo se situe au carrefour de différentes logiques ludiques: d'une part l'utilisateur cherche à stimuler à nouveau des effets de plaisir expérimenté en situation, d'autre part le 
logiciel permet de rendre cette création amusante dans ses logiques propres. Ces dynamiques dialoguent en partie, lorsque des possibilités du logiciel font référence explicitement à des expériences de jeu.

\section{Le public est secondaire : I' ancrage dans le jeu par la gratuité de l'acte}

Selon Manuel Boutet, un même jeu vidéo peut être joué de différentes manières et les joueurs développent des «styles de jeu » personnels qui «se forment dans la recherche d'une situation qui vaut d'être répétée - autrement dit, ils émergent de l'exploration pratique de tout ce qui fait un bon moment, de tout ce qui le prolonge ou le nourrit. » (Boutet, 2012, p. 212) Parmi les joueurs de RPG, par exemple, se trouvent des personnes ayant des définitions différentes de ce qui fait un bon moment : certains portent une attention toute particulière à la narration quand d'autres préfèrent la finesse du système de combats. Chacun développe un style de jeu qui, dans un premier temps, peut rester indicible pour le chercheur :

Ensuite seulement, ce qu'un style porte d'ancrages locaux et d'ajustements créatifs peut le rendre saillant en public, c'est-à-dire en dehors du milieu où il s'est formé, comme manifestation d'une autre forme d'existence possible. C'est souvent à ce moment-là aussi qu'il devient repérable pour le sociologue. (Boutet, 2012, p. 212)

Les créateurs développant des œuvres dans lesquels ils expriment leurs goûts, nous retrouvons dans leurs jeux l'expression d'un style de jeu personnel. Les enquêtés matérialisent leurs préférences en tant que joueurs dans leurs créations. Lorsqu'ils rendent leurs productions publiques, il arrive qu'ils aient à se confronter aux styles de jeux différents. Sur un forum dédié à des fangames de Zelda, un utilisateur de Zquest (logiciel de création de jeux Zelda) alimente irrégulièrement un topic de discussion dans lequel il publie des nouvelles sur le développement de son jeu. Il y explique notamment que dans son Zelda, les objets (équipements des personnages, etc.) occupent une place importante et que la difficulté y est élevée. D'autres amateurs interviennent alors pour tenter d'infléchir ses choix en le rappelant à la norme : "ce n'est plus un Zelda avec autant d'objets! », ou en se référant au public potentiel : "la difficulté trop dure va rebuter le public plus jeune ». L'auteur y répond en expliquant que c'est «[son] Zelda » et qu'en l'occurrence il continuera de faire ce qui lui plait, à développer ce que lui préfère dans ces jeux.

Dans ces conditions, les jeux qui sont publiés sur les forums peuvent être envisagés comme la "photographie sédimentée " (Hennion, 2009, p. 71) des goûts de leurs auteurs, tout en étant en partie des constructions sociales permettant d'exhiber le capital technique et culturel de leurs auteurs. C'est un processus que l'on peut sans doute retrouver dans la production commerciale (voir à ce sujet l'article de Charrieras et Roy-Valex, 2015), mais probablement pas de manière aussi centrale. Un professionnel d'un studio belge nous expliquait lors d'une rencontre entre développeurs que, pour lui, «le joueur a toujours raison» et que les séances de playtests guident fortement l'évolution du projet au cours du temps dans les studios de développement. Sur le marché, le produit doit trouver son public. Au contraire, dans les communautés amateurs, la démarche est avant tout personnelle (on agit "par passion », " pour soi-même ») et ensuite le public est en partie déjà constitué : les jeux 
créés le sont à destination de la communauté dans laquelle on intervient. Cela n'exclut pas des succès plus larges, y compris quand ils sont accidentels.

Le cas de Marc est éclairant à ce sujet. Il explique avoir, dans un premier temps, utilisé RPG Maker pour faire des petits RPGs classiques au début de son adolescence: "des trucs avec des dragons et des émeraudes ", dit-il en plaisantant. Après avoir fait une pause pendant quelques temps, il décide de créer un jeu "de manière super impulsive ", "juste comme ça, pour voir », en partie parce qu'il cherche à "voir si [il est] meilleur qu'avant " sur ce logiciel. Il achève sa création en un an, un jeu qui s'éloigne des codes des RPGs classiques sur le plan narratif et « super bâclé » selon lui. Il poste sa création sur un site phare parmi les communautés de RPG Maker. Il reçoit quelques dizaines de commentaires ainsi qu'un prix honorifique de la communauté qui distingue les meilleurs jeux. Il est «déjà super content comme ça, [il se dit] que c'est bon comme ça, quoi »: le succès atteint les proportions attendues et il peut passer à autre chose.

Deux ans plus tard, une personne lui demande s'il peut traduire son jeu en anglais, ce qu'il accepte. De fil en aiguille, sa création va être chroniquée sur un blog d'un célèbre game designer indépendant et par des youtubeurs influents. Le jeu sera ensuite traduit en espagnol, portugais et japonais. Dans des conventions aux États-Unis, des fans réalisent des costumes (cosplays) de ses personnages. Lorsqu'il nous explique cela, l'enquêté semble mal-à-l'aise : "c'était super bizarre », «ce n'est pas du tout dans cette optique que je l'ai fait ». Il affirme aussi que « tous les gens qui trouvent le jeu génial, c'est qu'ils ne connaissent pas les jeux auxquels j'ai joué [et auxquels] j'ai tout piqué». Par exemple, il s'est inspiré d'un élément de Metal Gear Solid pour réaliser une séquence de sa production où il demande au joueur de trouver un code secret dans un fichier texte livré avec sa création. Lors de l'entretien, comme dans les descriptions qu'il fait de son jeu en ligne, l'enquêté assume et revendique cette intertextualité, ses influences et ses hommages : il partage ses goûts. Tout développeur cherchant à " vivre de sa passion " aurait capitalisé sur ce succès inattendu. Lui se limite à vendre quelques artworks lorsqu'il a des problèmes d'argent.

Ce cas de succès accidentel démontre que la première adresse de l'amateur est soimême: notre enquêté cherchait à vérifier s'il était "devenu meilleur» dans l'utilisation du logiciel et voulait «voir » s'il pouvait faire un bon jeu vidéo. L'adresse secondaire est le groupe déjà constitué et identifié que forme la communauté et qui comprend les logiques qui sous-tendent cette création, notamment l'intertextualité et la gratuité de l'acte. Un succès au-delà de cette frontière peut être source de malaise, l'enquêté semblant redouter d'être désigné comme un imposteur. On peut voir dans cette importance de la gratuité de l'acte une forme d'ancrage dans le jeu, réputé improductif (Caillois, 1992).

\section{Du plaisir de joueur à celui de créateur : l'émancipation de la création}

31 Sur un forum important consacré à RPG Maker, un débat a débuté vers 2006-2007 lorsque l'équipe du site a voulu distinguer les «meilleurs jeux » issus de la communauté (le débat a perduré à travers le temps mais sous diverses formes et généralement de manière moins radicale). Les différends entre les «staffeux » (tels que sont désignés les membres qui participent à l'équipe rédactionnelle du site) ont révélé une fracture entre 
deux positionnements. Un premier groupe préférait mettre en avant une utilisation "classique » de RPG Maker et valoriser les RPGs traditionnels (longue durée de vie, quête épique, grand nombre d'objets, quêtes annexes nombreuses, etc.). Un deuxième groupe voulait quant à lui récompenser les jeux "originaux»: ces utilisateurs mettaient en avant la liberté des "amateurs" de proposer autre chose et de se distinguer des jeux traditionnels, notamment en « tordant » le logiciel pour réaliser des choses inattendues. On retrouve ici une fracture déjà documentée dans le champ du cinéma amateur: il y a d'une part les amateurs qui imitent la "grammaire vidéoludique » - nous paraphrasons Roger Odin (1999) lorsqu'il décrit le respect de la grammaire cinématographique dans le cinéma amateur - et ceux qui cherchent à s'en éloigner. Par ailleurs, les tenants du classicisme défendaient un recours assumé aux images de base fournies avec le logiciel, alors que les tenants de l'originalité préféraient l'apport de dessins personnels.

Une des caractéristiques de ce débat est qu'il repose en partie sur les plaisirs développés dans le cadre de la création en elle-même. Deux entretiens de personnes ayant pris part à ce débat, un tenant de l'originalité et un tenant du classicisme, sont éclairants à ce sujet. Chacun d'eux en est venu à utiliser RPG Maker pour créer des RPG classiques: Samuel voulait créer une suite de son jeu préféré, Yann souhaitait continuer des « sortes de jeux de rôle papier » qu'il faisait étant enfant en s'inspirant de jeux commerciaux.

33 Cependant, au cours du temps, le premier va «laisser tomber l'idée de faire des vrais jeux » avec le logiciel afin de "tester ", " expérimenter ». Il crée alors toute une série de «trucs» très courts. Sachant dessiner, il peut produire des œuvres «full custom " (entièrement faits main sur le plan graphique). Au contraire, Yann, le tenant des jeux classiques, ne fera que pousser plus loin ses critères de qualité, notamment celui de l'écriture d'une histoire solide. Il se décrit comme accordant plus "d'importance au fond qu'à la forme » pour expliquer qu'il ne porte pas une attention particulière aux graphismes ("tant que c'est bien mappé »: c'est-à-dire tant que les tuiles graphiques sont assemblées de manière cohérente et harmonieuse). Dessiner ne fait pas partie de sa définition d'un bon moment de création. Préférant par-dessus tout écrire des scénarios longs et des retournements de situation, l'enquêté va préférer produire quelques jeux très longs.

Ici aussi, l'utilisateur explore " ce qui fait un bon moment » et cherche à le prolonger en répétant dans le temps son activité. La particularité de cette étape est qu'il y a à nouveau une mutation ou un déplacement du plaisir du joueur, mouvement cette foisci caractérisée par une émancipation de la création par rapport au jeu initial. Bien que l'activité soit toujours référentielle - elle le reste indéfiniment dans de nombreux cas une dimension supplémentaire vient s'y superposer. Le plaisir qui est développé l'est à partir du dispositif de création lui-même, dans son exploration, son utilisation répétée, voire sa maitrise, peut-être sa personnalisation.

35 Il ne s'agit pas de se soumettre à la logique du logiciel, mais de l'explorer jusqu'à l'établissement de pratiques jugées plaisantes pour soi. Ainsi Samuel détourne progressivement le logiciel pour créer de petits jeux expérimentaux, tandis que Yann développe des routines d'écriture de scénario et de "mapping ». Au «style de jeu », qui décrit la recherche du plaisir vidéoludique, nous proposons donc d'ajouter un « style de création ludique » pour nommer cette quête du plaisir de la création et du maniement des logiciels. 


\section{Quand ce n'est plus du jeu : la lassitude, la professionnalisation et l'auctorialisation}

Lorsqu'on demande à un enquêté (lors des entretiens mais aussi lorsqu'ils discutent entre eux sur les forums à propos de l'étiquette d'amateur) s'il se reconnaît dans le terme "amateur", deux réactions sont possibles. Il y a ceux qui revendiquent leur amateurisme, qui le portent avec sourire : « oui je fais ça uniquement pour le fun ». Et puis il y a ceux qui doivent l'admettre, le concéder : tant qu'ils ne gagnent pas leur vie grâce à leurs jeux, alors oui, ils sont des amateurs. Ils préfèrent souvent s'agglomérer aux « indés ", dont on ne précise communément pas la situation professionnelle. C'est la définition de l'amateur par la négative : celui qui n'est pas (encore) professionnel, mais qui cherche à le devenir. Ceux-là agissent alors en suivant leur représentation de ce qu'un professionnel devrait faire : analyser le marché, trouver une niche, réaliser le jeu, intervenir sur les communautés dans le but de créer une émulation autour de leurs créations, essayer de penser autant au jeu qu'à la façon de le vendre. Ils semblent se détacher de la sphère ludique à proprement parler: ils s'organisent là où d'autres improvisent, et la productivité de l'activité prime sur le plaisir.

deuxième forme de sortie d'une dynamique strictement ludique observée est l'auctorialisation, la construction d'une identité d'auteur. Il s'agit de créateurs qui, souvent après des années de pratique, se permettent d'avoir un projet d'auteur, une intention artistique, avec une idée qui "vaut le coup» de finir le jeu en cours de création. Cette dynamique est présente en germe - comme horizon d'attente? - chez tous nos enquêtés qui nous affirment l'éventualité, « un jour ", de créer un "vrai » jeu, lorsqu'ils auront «trouvé une vraie bonne idée ». Un «vrai jeu» vient nommer une distinction de légitimé - y compris dans le discours des créateurs - entre les produits payants, créés par des professionnels ou des artistes et « vus dans Joypad» et les « petits jeux », les « trucs » gratuits, créés dans le seul cadre passionnel de l'amateurisme.

On retrouve, dans ces deux cas, la distinction vue plus haut entre les usages ludiques et les usages "sérieux " de RPG Maker, par exemple. L'usage "sérieux » est celui du professionnel (y compris en devenir) qui compte «faire ce que le public veut », etc. A une époque, sur certains forums de RPG Maker, plusieurs créateurs se sont prononcés pour que les jeux soient payants. En retour, les amateurs opposés à cette approche dénonçaient un virage "serious business" des communautés. Il s'agit là d'un renversement important dans la démarche de l'amateur, qui passe d'une création dont il est la principale adresse et centrée sur ses goûts, à une démarche orientée vers le goût imaginé d'une future audience.

Parmi les facteurs explicatifs d'un tel renversement, les enquêtés évoquent l'évolution du marché, l'apparition de plateformes de vente comme Steam rendant la commercialisation de jeux amateurs bien plus aisée. Il y a donc probablement des enjeux à soulever en termes de configuration du champ dans le temps. Cette distinction en termes d'usages dits sérieux ou non nous permet d'affiner les modalités de la construction de la relation usagers-logiciels. Là où l'amateur assumé se laissera surprendre par le logiciel de création, en étant amusé d'une réaction inattendue, le (futur) professionnel aura tendance à y voir une erreur à corriger. 
40 La dynamique de lassitude est peut-être plus commune, quoique plus difficile à identifier et à recenser. Stéphane crée son propre jeu tout seul depuis plus de dix ans. Il veut finir cette œuvre «parce que c'est vraiment $l e$ jeu qu'[il] voulait faire » mais admet que "ça commence à faire long ", que ce n'est "plus très marrant ». Il est d'ailleurs révélateur que Stéphane ne souhaite pas faire «trop» de publicité en dehors de sa communauté, car les joueurs extérieurs «alors même que c'est un jeu fait sur le temps libre » se montrent très exigeants et répondent surtout par de froids rapports de bugs. « Du coup je ne fais plus de pub, dit-il, parce que je n'ai pas envie de faire de débogage pendant une semaine ». Les créateurs ne sont pas condamnés à arriver à ce stade de la lassitude, mais ce phénomène permet de remettre en perspective les trajectoires des acteurs. Les dynamiques de professionnalisation, d'auctorialisation ou de lassitude viennent « corrompre » le jeu (Caillois 1992).

\section{Conclusion : le passage d'amateur-joueur à amateur- créateur}

41 A travers l'exposition des premiers résultats de notre étude en cours, nous avons cherché à interroger les liens entre jeu et création comme autant de passages. Pour expliquer comment nos enquêtés sont passés de joueur à créateur, comment ils ont basculé, nous avons proposé une série de dynamiques: le prolongement du jeu, l'appropriation ludique de moyens de création, le déplacement de la stimulation du plaisir, l'ancrage dans la gratuité, l'émancipation de la création et enfin les possibles sorties de la sphère ludique (lassitude, auctorialisation et professionnalisme). Ces dynamiques ne sont ni exhaustives ni hermétiques et il nous faudra, dans de futures recherches, étudier quels rapports elles entretiennent.

42 En cherchant à étudier le changement de posture de nos enquêtés, c'est finalement une constante qui émerge en creux. Quand le joueur s'arrête de jouer pour créer, il semble continuer à se positionner du point de vue de la réception. Nous sommes alors tentés de dire qu'il agit « en tant que joueur». C'est que le terme de « joueur» est trompeur : en ne désignant l'acteur que par sa pratique, nous désignons pêle-mêle le joueur passionné et le joueur occasionnel, le joueur effectif et le joueur déclaré. Dire que les enquêtés agissent « en tant que joueur » ne permet pas de déterminer ce que cela implique. C'est pourquoi nous proposons de remettre le terme d' " amateur » au cœur de l'explication. En effet, ces créateurs "amateurs» au sens économique du terme (en dehors des circuits commerciaux) agissent avant tout comme des " amateurs de » jeux vidéo. C'est cette notion, généralement utilisée assez naïvement dans le champ des sciences du jeu, qui nous permet l'articulation théorique manquante dans de nombreux travaux.

43 Antoine Hennion (2009) entend mettre « la réflexivité du côté des amateurs » : le goût de l'amateur est issu d'un effort explicite, d'une intention, d'une volonté et est surtout un processus réflexif.

Le goût, le plaisir, l'effet ne sont pas des variables exogènes, ou des attributs automatiques des objets. Ils sont le résultat d'une pratique corporelle, collective et instrumentée, réglée par des méthodes elles-mêmes sans arrêt rediscutées, orientées autour de la saisie appropriée d'effets incertains (...) (Hennion, 2009, p. 67).

Nous proposons de parler non pas d'un passage de joueur à créateur mais d'amateurjoueur à amateur-créateur. 

du goût serait profitable pour l'ensemble des études des pratiques créatives liées au jeu vidéo. Les magazines spécialisés du jeu vidéo ne cherchent-ils pas à travailler indirectement le goût de l'amateur? Les spectateurs de Let's Play, de speed runs, voire de certains machinimas ne sont-ils pas à envisager comme autant d'amateurs de jeux vidéo, produisant du «plaisir de jeu » de manière secondaire ? Nous empruntons le terme à Julie Delbouille (2016) qui parle de "joueur secondaire» pour désigner le statut et l'activité du spectateur d'une situation de jeu en faisant référence à James Newman (2002)

De plus, cette mise en lumière du processus réflexif de l'amateur pourrait permettre de contrer les approches du virtuel comme un domaine « immersif » dans lequel le joueur plongerait, voire pourrait se perdre. Le joueur-amateur travaille ses goûts en relation aux signes, aux dispositifs et au contexte. Se passer de la notion d' " amateur de jeu 
vidéo » et se limiter aux usages indifférenciés du terme « joueur » reviendrait à étudier les cinéphiles en tant que spectateurs ou les amateurs de musique en tant qu'auditeurs. La reconnaissance du statut d'amateur de jeux vidéo (ou de «ludophile»?) pourrait certainement aider à légitimer le média comme pratique culturelle.

Enfin, il nous faut terminer en affirmant les limites de cette étude. Il y a bien d'autres dynamiques qui entrent en jeu dans la pratique de création en amateur de jeux vidéo, tels que les dynamiques sociales, le processus de remix d'éléments depuis des bases de données ou encore la recherche d'une expression personnelle. Et puis, comme nous l'avons entraperçu, toutes ces dynamiques interagissent et se définissent respectivement. Il ne s'agit pas pour nous de dire que la création en amateur n'est qu'une façon de produire autrement du plaisir de jeu. Mais il n'en demeure pas moins que c'est une dimension importante pour comprendre cette pratique encore peu documentée, qui met par ailleurs en évidence l'opportunité pour les études du jeu d'aller puiser dans les outils théoriques des études des pratiques culturelles.

\section{BIBLIOGRAPHIE}

BURKE Q et KAFAI Y.B. (2014), « Decade of Game Making for Learning: From Tools to Communities », in M.C. Angelides et H. Agius (eds.), Handbook of Digital Games, Hoboken, USA, John Wiley \& Sons, pp. 689-709.

BOUTET M. (2012), « Jouer aux jeux vidéo avec style. Pour une ethnographie des sociabilités vidéoludiques », Réseaux, n²07, pp.173-174.

CAILLOIS R. (1992 [1958]), Les Jeux et les hommes, Paris, Gallimard.

CHARRIERAS D. et ROY-VALEX M. (2015), « Culture de la convergence chez les créatifs de jeux vidéo », Communication [En ligne], vol.33/2, http://communication.revues.org/5976

DELBOUILLE J (2016), « L'avatar vidéoludique : retour sur les notions d'immersion et de joueur secondaire », Séminaire du Liège Gamelab, 5 octobre 2016.

DJAOUTI D. (2011), « Serious Game Design: considérations théoriques et techniques sur la création de jeux vidéo à vocation utilitaire, thèse de doctorat, Université de Toulouse.

DUBOIS V, MEON J.M., PIERRU E. (2010), « Quand le goût ne fait pas la pratique. Les musiciens amateurs des orchestres d'harmonie ", Actes de la recherche en sciences sociales, $\mathrm{n}^{\circ} 181-182, \mathrm{pp}$. 106-125.

FLICHY P. (2010), Le sacre de l'amateur: sociologie des passions ordinaires à l'ère numérique, Paris, Seuil.

GEE.R. et TRAN K.M. (2016), « Video Game Making and Modding », in B. Guzztti et M. Lesley (eds.), Handbook of Research on the Societal Impact of Digital Media, Hershley, USA, Information Science Reference, pp. 238-67.

GENVO S. (2012), « La théorie de la ludicisation : une approche anti-essentialiste des phénomènes ludiques », Communication lors de la journée d'études Jeu et jouabilité à l'ère numérique, le 8 
décembre 2012, organisée par l'Université Paris 1 Panthéon Sorbonne, à l'Institut d'Arts et d'Archéologie, Paris.

HEAS S. et POUTRAIN V. (2003), « Les méthodes d'enquête qualitative sur Internet », ethnographiques.org, ${ }^{\circ} 4$, http://www.ethnographiques.org/2003/Heas,Poutrain

HENNION A. (2009), « Réflexivités. L'activité de l'amateur », Réseaux, n¹53, pp. 55-78.

JENKINS H. (2006), Convergence culture: where old and new media collide, New York, NY University Press.

NEWMAN J. (2002), « The Myth of the Ergodic Videogame. Some toughts on player-character relationships in videogames ", Game Studies, vol. 2(1), pp. 1-8.

ODIN R. (1999), « La question de l'amateur », Communications, n68, pp. 47-89.

OWENS T. (2013), « Mr. Moo's First RPG: Rules, Discussion and the Instructional Implications of Collective Intelligence on the Open Web ", in M. S. S. Johnson et R. S. Colby (eds.), Rhetoric/ Composition/Play: How Electronic Games Mediate Composition Theory and Practice (and Vice Versa), New York, Palgrave Macmillan, pp. 193-204.

POSTIGO H. (2007), « Of mods and modders: Chasing down the value of fan-based digital game modifications ", Games and Culture, n², pp. 300-313.

ROTH M.E. (2015), « At the Edge of a 'Digital Area' - Locating Small-Scale Game Creation », Asiascape: Digital Asia, n², pp. 183-212.

SHAW A. (2010), « What Is Video Game Culture? Cultural Studies and Game », Games and Culture, $\mathrm{n}^{\circ} 5$, pp. 403-424.

SCHAEFFER J.M. (1999), Pourquoi la fiction?, Paris, Seuil.

SOTAMAA O. (2010), « When the Game Is Not Enough: Motivations and Practices Among Computer Game Modding Culture », Games and Culture, n5, pp. 239-255.

\section{NOTES}

1. «Simple enough for a child, powerful enough for a game developer. »

\section{RÉSUMÉS}

La création de jeux vidéo en amateur à l'aide de logiciels (Unity, Construct 2, RPG Maker, etc.) a été peu étudiée. Pourtant, cette pratique importante sur le plan quantitatif et hétérogène sur le plan qualitatif permet notamment d'interroger une des marges du jeu: celle de la création. Comment les joueurs passent-ils à la création? S'agit-il d'un prolongement du jeu? Et si oui, qu'est-ce qui est prolongé dans ce cas? Ce sont ces questions que nous abordons dans cet article, sur la base des premiers résultats d'une enquête ethnographique en cours. Nous mettons d'abord à jour plusieurs dynamiques caractérisant l'activité de ces créateurs. Puis, en nous fondant sur ces résultats et en introduisant la notion d'amateurisme dans le champ des sciences du jeu, nous 
proposons de problématiser la question du prolongement ludique au regard de l'activité réflexive que constitue le goût.

The field of amateurs using game creation tools (Unity, Construct 2, RPG Maker, etc.) has been, up to now, little explored. Yet, the activity is important quantitatively and heterogeneous qualitatively. And, above all, it allows to study one of the play's margins, namely creation. How do tool users move from players to makers? Is this process an extension of playing itself? If so, what exactly is extended? We address these questions in this paper, presenting the first results of our still ongoing ethnographic fieldwork. Firstly, we name various dynamics characterizing the makers' activity. Then, on this basis and reintroducing the notion of hobbyism in the field of game studies, we propose to problematize the question of the playing extension as a taste related reflexive activity.

INDEX

Mots-clés : amateur, fan, game design, logiciels, jeu, attitude ludique

Keywords : amateur, fan, game design, programs, tools, play, playfulness

\section{AUTEUR}

PIERRE-YVES HUREL

Liège Gamelab (Lemme) - Université de Liège 